\title{
Slow waves in microchannel metal waveguides and application to particle acceleration
}

\author{
L. C. Steinhauer \\ Redmond Plasma Physics Laboratory, University of Washington, \\ 14700 NE 95th Street, Suite 100, Redmond, Washington 98052, USA \\ W. D. Kimura* \\ STI Optronics, Inc., 2755 Northup Way, Bellevue, Washington 98004, USA
}

(Received 10 April 2003; published 9 June 2003)

\begin{abstract}
Conventional metal-wall waveguides support waveguide modes with phase velocities exceeding the speed of light. However, for infrared frequencies and guide dimensions of a fraction of a millimeter, one of the waveguide modes can have a phase velocity equal to or less than the speed of light. Such a metal microchannel then acts as a slow-wave structure. Furthermore, if it is a transverse magnetic mode, the electric field has a component along the direction of propagation. Therefore, a strong exchange of energy can occur between a beam of charged particles and this slow-waveguide mode. Moreover, the energy exchange can be sustained over a distance limited only by the natural damping of the wave. This makes the microchannel metal waveguide an attractive possibility for high-gradient electron laser acceleration because the wave can be directly energized by a long-wavelength laser. Indeed the frequency of $\mathrm{CO}_{2}$ lasers lies at a fortuitous wavelength that produces a strong laser-particle interaction in a channel of reasonable macroscopic size (e.g., $\sim 0.6 \mathrm{~mm}$ ). The dispersion properties including phase velocity and damping for the slow wave are developed. The performance and other issues related to laser accelerator applications are discussed.
\end{abstract}

DOI: 10.1103/PhysRevSTAB.6.061302

PACS numbers: 41.75.Jv, 42.55.Lt

\section{INTRODUCTION}

A conventional metal-wall waveguide supports a series of waveguide modes. One class of these is transverse magnetic (TM) waves, which have a forward component of the electric field. This field component could be used for energy exchange with a beam of particles except for the fact that these are "fast" waves with a phase velocity exceeding $c$, the speed of light in vacuum. Thus charged particles, even traveling at $\sim c$, rapidly slip out of phase with the wave. However, under special conditions (e.g., proper frequency and channel size) a metal waveguide supports one slow-wave mode that can have a phase velocity equal to or less than $c$. The slow-wave mode appears only because of the negative dielectric function in a metal. What makes the slow wave particularly interesting is that for "microchannel" sizes (i.e., waveguides 100 's of $\mu \mathrm{m}$ in diameter) the slow-wave frequency is in the infrared range. Such a slow wave may be directly energized by long-wavelength laser light with the proper polarization. For example, in a cylindrical copper guide with $0.6-\mathrm{mm}$ diameter, the slow wave can be directly driven by a $\mathrm{CO}_{2}$ laser $(\lambda=10.6 \mu \mathrm{m})$. The laser light is focused into one end of the guide and propagates along it in a slow-wave TM mode. Figure 1 illustrates how this arrangement can be used for charged particle acceleration $[1,2]$. As an aside, we also note this same mechanism can

\footnotetext{
*Corresponding author.

Email address: wkimura@stioptronics.com
}

be used for the inverse process whereby coherent light is extracted from a charged particle beam. However, this inverse process will not be discussed in this paper.

The outline of this paper is as follows. Section II develops the dispersion relation in metal waveguides. The conditions under which the slow-waveguide mode can appear are identified. Its dispersion properties including phase velocity and damping are found. Section III focuses on the application of slow-waveguide modes to accelerators. This begins with a discussion of where this approach fits within the family of accelerator concepts. The predicted acceleration performance of the slow wave is considered next followed by a comparison with another advanced accelerator concept, the laser wakefield accelerator (LWFA). Section IV concludes the paper with a discussion of issues related to the metal microchannel concept that are appropriate for future investigations.

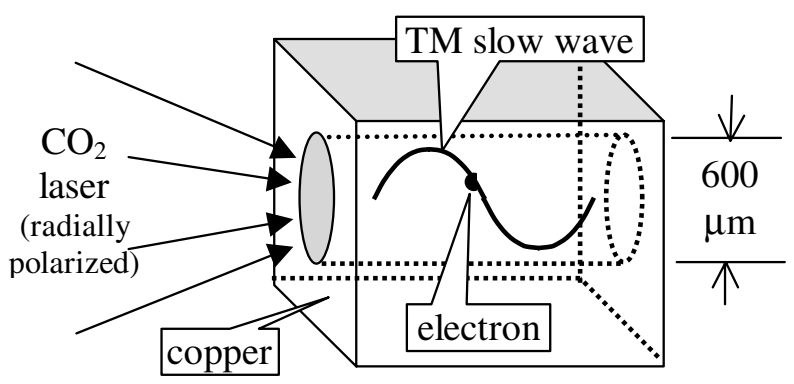

FIG. 1. Laser-particle acceleration in a metal microchannel. 


\section{METAL WAVEGUIDE DISPERSION RELATION}

\section{A. Electromagnetic waves in simple two-medium structures}

Consider the propagation of waves in a two-medium structure with wave vectors tangential to the interface between the media. The most familiar example of this is a waveguide, as shown in Fig. 2(a), composed of a channel medium (1) sandwiched between two sections of boundary medium (2). The figure illustrates both the transverse structure of a waveguide wave (dark line) and its propagation along the guide. The figure portrays a slab guide (planar sides), but the guide might be cylindrical (hollow tube), rectangular (rectangular or square tube), or have a more complicated cross section.

Waves in waveguides appear as a discrete series of eigenmodes, each with its own characteristics (i.e., wave number, transverse structure). Common waveguide modes of interest have a transverse field structure that is oscillatory in the channel (sideways component of the wave vector $k_{\perp}$ ) and evanescent at the boundary. In the channel, there is in effect a transverse standing wave, which is the sum of a "forward" traveling wave $\left(+k_{\perp}\right)$ and a "reverse" traveling wave $\left(-k_{\perp}\right)$. The oscillatory structure in the guide [dark line in Fig. 2(a)] is the standing wave pattern. Depending on the mode number there can be any number of oscillatory lobes in the channel. An important feature of waveguide modes when the channel medium (1) is a vacuum is that their phase velocity $\omega / k$ exceeds the speed of light $c(\omega=$ frequency, $k=$ wave number $=2 \pi / \lambda$ ).

Under certain conditions (e.g., frequency, dielectric constants of the media, channel width) one of the waveguide modes can have the unusual property that the transverse component of the wave vector in the channel is imaginary, $k_{\perp}=i\left|k_{\perp}\right|$. In this case the transverse structure in the channel is also evanescent, as illustrated

(a) Standard waveguide.

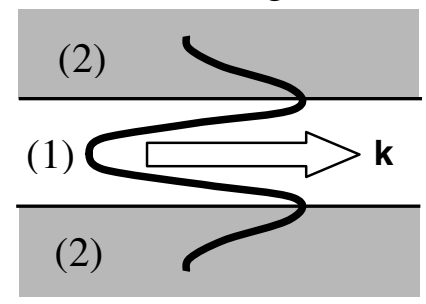

(b) Special waveguide mode.

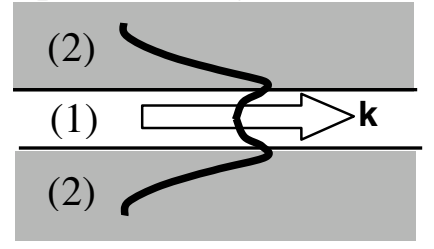

FIG. 2. Waveguide mode types. in Fig. 2(b). In the example here the two interfaces are close enough that the field strength in the center of the channel is still a large fraction of its maximum value, which is at the surfaces. A profound consequence of imaginary $k_{\perp}$ is that in a vacuum channel the phase velocity of the wave $\omega / k$ can be less than or equal to $c$. Thus, this special eigenmode might be called the slowwaveguide mode. The synchronous case $\omega / k=c$ is of special interest because a relativistic charged particle moving at $c$ is exactly synchronous with the slow wave and can be accelerated over distances vastly longer than the Rayleigh length. The slow-waveguide mode has been analyzed in the context of a plasma channel (where region 2 is a plasma) in Ref. [3], where it is called a "channel wave."

The slow-waveguide mode [Fig. 2(b)] is closely related to another phenomenon called surface waves. The same conditions that generate a slow wave in a waveguide can also produce surface waves at a single interface. This wave is evanescent in both directions away from the interface. In effect then, the slow-waveguide mode can be viewed as a pair of overlapped opposing surface waves.

Slow waves are well known in the accelerator community. However, they typically require slow-wave structures in which disks, irises, or other obstructions are inserted into a waveguide, or the guide wall is given a wavy structure. The appeal of the slow-waveguide mode discussed here is that no such artificial structures are needed. A simple waveguide (albeit with a particular size and dielectric constant) is all that is required. This slow-waveguide mode travels on the simplest of all possible slow-wave structures - a smooth waveguide.

In the next sections, the conditions that allow a slowwaveguide mode are found. After establishing the basic equations, surface waves are considered first because they exhibit some features of the slow-waveguide mode, but their analysis is simpler. Attention then turns to the slowwaveguide mode in slab and cylindrical guides. Special attention will be given to the case of a vacuum channel and a metal or plasma boundary.

\section{B. Basic field equations}

For the surface wave or slab channel we adopt a Cartesian coordinate system where $z$ is the direction of propagation and $x$ is the coordinate perpendicular to the interface(s). For a surface wave the interface is at $x=0$. For the slab guide the interfaces are at $x= \pm b$, and the center of the channel is at $x=0$. For the cylindrical guide we adopt a cylindrical coordinate system where $z$ is the direction of propagation and $r$ is the coordinate perpendicular to the interface at $r=b$. The center of the channel is at $r=0$.

Nonevolving waves in these guides have the form $\mathbf{E}=$ $\hat{\mathbf{E}}(r) \exp [i(k z-\omega t)]$, where the real part is understood. Here $\omega$ is the wave frequency and $k$ is its propagation 
constant. In the slab guide $r$ is replaced by $x$. The primary interest is in waves with a forward electric field component on the axis. In waveguide parlance these are TM waves since the magnetic field is purely in the $\theta$ direction ( $y$ direction in the slab case). With $\mathbf{E} \sim e^{-i \omega t}$, the wave propagation is governed by Maxwell's equation $\left[\nabla^{2}+\right.$ $\left.\varepsilon(\omega / c)^{2}\right] \mathbf{E}=0$, where $\varepsilon(\omega)$ is the dielectric function. The longitudinal electric field in a cylindrical guide is governed by

$$
\begin{gathered}
{\left[\frac{1}{r} \frac{d}{d r}\left(r \frac{d}{d r}\right)-\alpha^{2}\right] \hat{E}_{z}=0,} \\
\alpha=\sqrt{k^{2}-\left(\omega^{2} / c^{2}\right) \varepsilon} .
\end{gathered}
$$

Transverse field components are related to the longitudinal component by $\boldsymbol{\nabla} \cdot \mathbf{E}=0$. (The analogous equation for a slab guide is similar. Appendix A gives the slabguide equivalents both here and for the other equations that follow.)

The transverse scale parameter $\alpha$ is closely related to the nature of the propagating mode. A familiar case, the standard waveguide mode, has imaginary $\alpha_{1}$ and real $\alpha_{2}$,

$$
\begin{array}{ll}
|r| \leq b: & \hat{E}_{z}=G_{1} I_{0}\left(\alpha_{1} r\right) ; \\
|r|>b: & \hat{E}_{z}=G_{2} K_{0}\left(\alpha_{2} r\right)
\end{array}
$$

where $G_{1}$ and $G_{2}$ are constants, and $I_{0}, I_{1}, K_{0}$, and $K_{1}$ are modified Bessel functions.

In the event of imaginary $\alpha_{1}$, the Bessel functions in Eq. (3a) convert as follows: $I_{0}\left(\alpha_{1} r\right) \rightarrow J_{0}\left(\left|\alpha_{1}\right| r\right)$ and $-i I_{1}\left(\alpha_{1} r\right) \rightarrow J_{1}\left(\left|\alpha_{1}\right| r\right)$. From Maxwell's equations, the solutions given by Eq. (3) must satisfy the continuity conditions at the interface $(r=b)$ between the channel and the boundary media, $\left[\varepsilon \hat{E}_{r}\right]=0$ and $\left[\hat{E}_{z}\right]=0$, where the square brackets denote the jump at the interface. Eliminating $G_{1}$ and $G_{2}$ results in

$$
\varepsilon_{1} \frac{I_{1}\left(\alpha_{1} b\right)}{\alpha_{1} b I_{0}\left(\alpha_{1} b\right)}=-\varepsilon_{2} \frac{K_{1}\left(\alpha_{2} b\right)}{\alpha_{2} b K_{0}\left(\alpha_{2} b\right)},
$$

with $\alpha_{1}$ and $\alpha_{2}$ given by Eq. (2). Equation (4) represents the dispersion relation since $\alpha=\alpha(\omega, k)$ and $\varepsilon=\varepsilon(\omega)$, i.e., it governs the relationship between $\omega$ and $k$. For imaginary $\alpha_{1}$ the fraction on the left side of Eq. (4) becomes $J_{1}\left(\left|\alpha_{1}\right| b\right) /\left[\left|\alpha_{1}\right| b J_{0}\left(\left|\alpha_{1}\right| b\right)\right]$. Equation (4) is the same as Eq. (9) in Ref. [3]. For a surface wave the dispersion relation is simpler: $\varepsilon_{1} / \alpha_{1}=-\varepsilon_{2} / \alpha_{2}$.

For the slow-waveguide mode [see Fig. 2(b)], the mode has evanescent fields in both directions away from the interface, i.e., both $\alpha_{1}$ and $\alpha_{2}$ are predominantly real. Inspection of the dispersion relation, Eq. (4), gives the necessary condition for such a mode to exist. (This is most clear in the case of purely real $\varepsilon$, i.e., lossless propagation with no damping of the mode.) The necessary condition is that the dielectric constants on the two sides of the interface must have opposite sign. Thus, for where the subscripts 1 and 2 refer to the regions depicted in Fig. 2(a). Imaginary $\alpha_{1}$ implies that the wave structure in the channel is oscillatory, as shown in Fig. 2(a), i.e., the transverse component of the wave vector $k_{\perp}$ is real. (Recall that $k_{\perp}=i \alpha$, so that imaginary $\alpha$ yields real $k_{\perp}$.) Real $\alpha_{2}$ implies that the wave in the boundary region is evanescent, i.e., nonpropagating. If instead of a guide with two sides, there is only a single boundary, then this is the familiar case of total reflection with both incident and reflected waves in region 1 and no transmitted waves in region 2.

However, unique behavior arises if both $\alpha_{1}$ and $\alpha_{2}$ are real, i.e., evanescent waves in both directions away from the interface, as illustrated by the special waveguide mode in Fig. 2. For real $\alpha_{1}$ (or predominantly real) and a vacuum channel $\left(\varepsilon_{1}=1\right)$, Eq. (2) implies that $\omega / k_{R} \leq$ $c\left(k_{R}\right.$ is the real part of $k$ ), i.e., the special waveguide mode is a slow-wave mode, with phase velocity equal to or below the speed of light.

\section{Slow-waveguide mode without damping}

The solutions to Eq. (1) for a cylindrical guide are

$$
\begin{gathered}
\hat{E}_{r}=-i\left(k / \alpha_{1}\right) G_{1} I_{1}\left(\alpha_{1} r\right), \\
\hat{E}_{r}=i\left(k / \alpha_{2}\right) G_{2} K_{1}\left(\alpha_{2} r\right),
\end{gathered}
$$

example, if there is a vacuum in the channel, $\varepsilon_{1}=1$, then the dielectric constant in the boundary $\varepsilon_{2}$ must be negative.

Negative dielectric constant occurs in plasmas above the critical density and in metals for optical and lower frequencies. In a metal the dielectric function is (Ref. [4])

$$
\varepsilon(\omega)=\varepsilon_{0}-\omega_{p}^{2} / \omega(\omega+i \gamma),
$$

where $\varepsilon_{0}(\approx 1)$ is the dipole's contribution, $\omega_{p}=$ $\left(4 \pi e^{2} n / m_{e}\right)^{1 / 2}$ is the plasma frequency based on the density of electrons in the conduction band, and $\gamma$ is the damping constant. Electrons in the conduction band are "free" electrons in the sense that they have zero resonance frequency. Noble metals, such as gold, silver, and copper, have a single conduction-band electron so that the density of these electrons is the same as the atomic density. In fully ionized plasmas $\varepsilon_{0}=1$ and $n$ is the full electron density. In this section we shall limit our attention to the undamped case $\gamma=0$. The damped case will be addressed in Sec. II D.

The dispersion relation, Eq. (4), is an eigenvalue equation with an infinite number of solutions. A more illuminating form of it employs the parameter $\mu \equiv-\left(a_{1} b\right)^{2}$, where

$$
\mu=\left(\omega^{2} / c^{2}-k^{2}\right) b^{2} .
$$

Equation (6) follows from Eq. (2). For a vacuum channel $\left(\varepsilon_{1}=1\right)$, observe that $\mu \geq 0$ gives normal waveguide 
modes with imaginary $\alpha_{1}$, and $\mu<0$ gives the slowwaveguide mode with real $\alpha_{1}$. In terms of $\mu$ the wave number is $k=\left(\omega^{2} / c^{2}-\mu / b^{2}\right)^{1 / 2}$. Using the dielectric function $\varepsilon_{2}$ from Eq. (5) (with $\gamma=0$ ), the dispersion relation Eq. (4) becomes

$$
F(\mu)=\left(\frac{\omega_{p 2}^{2}}{\omega^{2}}-1\right) \frac{K_{1}\left(\sqrt{k_{p 2}^{2} b^{2}-\mu}\right)}{\sqrt{k_{p 2}^{2} b^{2}-\mu} \cdot K_{0}\left(\sqrt{k_{p 2}^{2} b^{2}-\mu}\right)},
$$

where $k_{p 2}=\omega_{p 2} / c$. The $F$ function here for a cylindrical guide is defined as

$$
F(\mu) \equiv\left\{\begin{array}{c}
J_{1}(\sqrt{\mu}) / \sqrt{\mu} J_{0}(\sqrt{\mu}) ; \quad \mu \geq 0, \\
I_{1}(\sqrt{-\mu}) / \sqrt{-\mu} I_{0}(\sqrt{-\mu}) ; \quad \mu<0
\end{array}\right\} .
$$

The dispersion relation Eq. (7) has an infinite number of discrete solutions for $\mu$, namely, the eigenvalues $\mu_{n}$, $n=1,2, \ldots$.

A waveguide mode is quite different from a surface wave. In a surface wave with a specified "drive" frequency $\omega$, there is a single propagating wave number $k$, except in the forbidden frequency gap in which no wave propagates. By contrast, the waveguide dispersion relation produces an infinite number of discrete solutions, one for each eigenvalue $\mu_{n}$, each of which corresponds to a particular value of $k$. Note that a surface wave with $\omega$ below the forbidden band is analogous to the slow-waveguide mode.

The nature of the solutions to the dispersion relation Eq. (7) can be seen by a graphical analysis. The eigenvalues are portrayed graphically in Fig. 3 for a cylindrical guide. The solid black lines are the $F(\mu)$ function on the left-hand side (LHS) of Eq. (7), where it exhibits an infinite series of singular points at $\mu \approx \pi^{2}(m-1 / 2)^{2}$, for $m=1,2, \ldots$ The right-hand side (RHS) of Eq. (7) can be approximated for a metal boundary with optical or lower frequencies, $\omega \ll \omega_{p 2}$. For low-index eigenvalues, $\mu=\mathrm{O}(1)$ and the expression $k_{p 2}^{2} b^{2}$ within the square

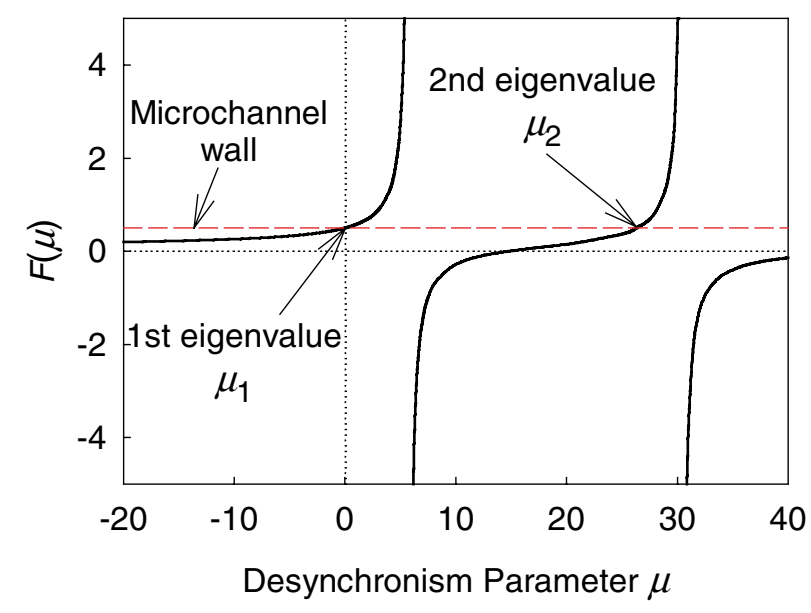

FIG. 3. (Color) Graphic portrayal of eigenvalues for cylindrical guide. roots on the RHS dominates. In this case, the RHS $\approx$ $k_{p 2} / k^{2} b=\lambda^{2} / 2 \pi \lambda_{p 2} b$, where the plasma wavelength in the metal is $\lambda_{p 2}=2 \pi / k_{p 2}$ and the wavelength is $\lambda=$ $2 \pi c / \omega$. [Note that for large arguments the factor on the RHS, $K_{1}(x) / K_{0}(x) \rightarrow 1$.] Thus the RHS is roughly independent of $\mu$ (since $\mu \ll k_{p 2}^{2} b^{2}$ ) and can be portrayed graphically as a horizontal red dashed line in Fig. 3.

Consider two examples:

(i) $X$-band radio frequencies $(\lambda \approx 33 \mathrm{~cm})$. Assume a copper guide diameter $2 b=4 \mathrm{~cm}$. Then the RHS $\approx 7 \times$ $10^{6}$. The eigenvalues occur wherever $F(\mu)$ equals this value (e.g., see the intersection of the solid and the dashed lines in Fig. 3). Since the RHS is so large, the intersections occur very close to the singularities of $F(\mu)$. The first eigenvalue is then $\mu_{1}=5.78$ and successive eigenvalues are approximately $\mu_{n} \approx \pi^{2}(n-1 / 2)^{2}$.

(ii) $\mathrm{CO}_{2}$ laser $(\lambda=10.6 \mu \mathrm{m})$. Assume a copper guide diameter $2 b=606 \mu \mathrm{m}$. Then the RHS $\approx 1 / 2$. This value is illustrated by the red dashed line in Fig. 3. Here the lowest eigenvalue (crossing point) is $\mu_{1}=0$. This gives a phase velocity of $c$, i.e., a slow-waveguide mode. Indeed this is the so-called synchronous case where the phase velocity of the wave exactly matches the speed of a highly relativistic particle.

Consider next the synchronous case. Setting the LHS = RHS, the dispersion relation can be expressed in the simple form

$$
F(\mu)=b_{\text {syn }} / 2 b,
$$

where the synchronous $(\omega / k=c)$ guide size in the undamped case is given by

$$
b_{\mathrm{syn} 0}=\lambda^{2} / \pi \lambda_{p 2} \text {. }
$$

For near synchronism (small $|\mu|$ ), the small-argument expansion of $F(\mu)$ can be used

$$
\frac{\omega^{2}}{c^{2}} \approx k^{2}+\frac{8}{b^{2}}\left(\frac{b_{\mathrm{syn} 0}}{b}-1\right) .
$$

Inspection of Eq. (11) shows that if $b>b_{\text {syn0 }}$, the phase velocity is slower than $c$, and if $b<b_{\text {syno }}$, the phase velocity is faster than $c$. The group velocity $v_{g} \equiv$ $d \omega / d k$ is slightly less than $c$. Using Eqs. (10) and (11) and assuming near-synchronous conditions, $b \approx b_{\text {syn } 0}$, the group velocity is $v_{g} \approx c\left(1-2 \lambda^{2} / \pi^{2} b^{2}\right)$.

Given the frequency $\omega$, the guide radius $b$, and the boundary material, the lowest eigenvalue $\mu_{1}$, and hence its wave number $k=k_{1}$, are determined in the manner described next. If the frequency is varied, then the relationship between $k_{1}$ on $\omega$ can be found, i.e., the dispersion curve. The dispersion curve has $\omega / k \approx c$ over a significant range of $k$, so it is helpful to plot the difference of the phase velocity from $c$, namely, $\omega / k-1$ vs $k$. Figure 4 shows this dependence for the lowest mode in cylindrical and slab guides, and for a surface wave. The guide examples assume a "microchannel" with diameter (width) 


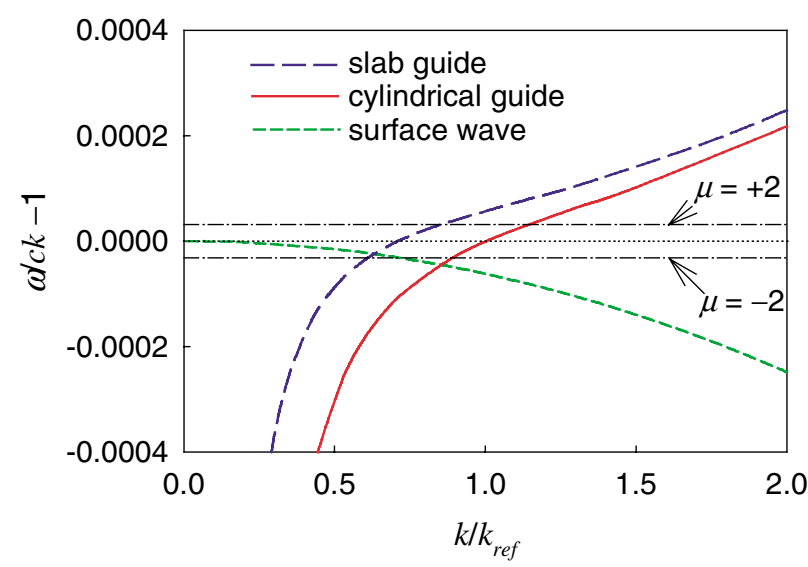

FIG. 4. (Color) Dispersion curves for various microchannel configurations. Plotted is relative phase velocity $\omega / c k-1$ vs wave number. The guide examples assume $b=300 \mu \mathrm{m}$. The reference wavelength $\lambda_{\text {ref }}=2 \pi / k_{\text {ref }}=10.6 \mu \mathrm{m}$.

$2 b=0.6 \mathrm{~mm}$. The points where the eigenvalue equals \pm 2 are marked with dashed horizontal lines. In the application to an accelerator, $|\omega / c k-1|$ must be small in order to avoid rapid dephasing of the wave relative to a charged particle moving at $c$. This and other accelerator-related questions are taken up in the next section.

Perfect synchronism $\omega / k=c$ occurs for a particular guide size given by Eq. (10). Table I compares the cylindrical guide sizes for a "standard" waveguide for the $X$ band and a synchronous waveguide. In the standard example, $\omega / k=\sqrt{2} c$ is assumed as being representative. Two laser frequencies are also shown. The standard waveguide diameters are comparable to the wavelength, whereas the synchronous guide diameters are usually much larger than a wavelength. The synchronous guide at $\mathrm{rf}$ frequencies is enormous and impractical. On the other hand, the short-wavelength laser example has a guide that is very tiny making its fabrication problematic. The infrared $\left(\mathrm{CO}_{2}\right)$ laser example is more interesting, with a small, but still macroscopic guide diameter of $\sim 0.6 \mathrm{~mm}$. For this example the group velocity is $v_{g}=$ $0.999747 c$, i.e., extremely close to $c$. Thus, the energy of the traveling wave moves at nearly the same speed as the relativistic particle beam.

\section{Slow-waveguide mode with damping}

Damping introduces an imaginary part to the dielectric function in the metal boundary. As a result, imagi- nary parts will appear in the eigenvalue $\mu$, the radial structure constants $\alpha_{1}$ and $\alpha_{2}$, and the propagation constant, $k$. The latter causes wave damping. This damping varies greatly from one metal to another. The usual expression for the dielectric constant in terms of the conductivity is $\varepsilon=\varepsilon_{0}+i 4 \pi \sigma / \omega$. Comparing this with Eq. (5), the conductivity is $\sigma=\omega_{p}^{2} / 4 \pi(\gamma-i \omega)$. The damping constant $\gamma$ can be inferred from conventional conductivity at low frequencies $(\omega \ll \gamma)$. Table II shows the damping constant for noble metals. These are factors of 4 or more lower than the $\mathrm{CO}_{2}$ laser frequency $\omega=$ $1.78 \times 10^{14} \mathrm{sec}^{-1}$.

For optical or lower frequencies and metals, $\omega_{p 2} \gg \omega$. The dielectric function Eq. (5) is then $\varepsilon_{2} \approx$ $-\left(\lambda / \lambda_{p 2}\right)^{2}(1+i \gamma / \omega)^{-1}$, where $\lambda_{p 2}=2 \pi c / \omega_{p 2}$ is the plasma wavelength in the boundary region. Since $\left|\varepsilon_{2}\right| \gg$ 1 , the radial structure constant Eq. (2) in the boundary region is $\alpha_{2} \approx(2 \pi / \lambda) \sqrt{-\varepsilon_{2}}$. Then the dispersion relation is

$$
F\left(\mu_{R}+i \mu_{I}\right)=\frac{b_{\mathrm{syn} 0}}{2 b} f_{1}(\gamma / \omega)\left[1-i f_{2}(\gamma / \omega)\right],
$$

where $\mu_{R}$ and $\mu_{I}$ are the real and imaginary parts of the eigenvalue, respectively,

$$
\begin{aligned}
& \mu_{R}=\left[\omega^{2} / c^{2} k_{R}^{2}-1+\left(k_{I} / k_{R}\right)^{2}\right] k_{R}^{2} b^{2}, \\
& \mu_{I}=-2\left(k_{I} / k_{R}\right) k_{R}^{2} b^{2},
\end{aligned}
$$

and the damping constant functions are defined as

$$
f_{1}(x) \equiv \sqrt{\frac{\sqrt{1+x^{2}}+1}{2\left(1+x^{2}\right)}}, \quad f_{2}(x) \equiv \sqrt{\frac{\sqrt{1+x^{2}}-1}{\sqrt{1+x^{2}}+1}} .
$$

The weak damping $(\gamma / \omega<1)$ limits of these functions are $f_{1} \approx 1-3 x^{2} / 8$ and $f_{2} \approx x / 2$. For the simplified treatment that follows in this section, we assume that the eigenvalue is small, $|\mu| \ll 1$. This allows the smallargument expansion to be used in Eq. (8), $F \approx(1 / 2)(1+$ $\mu / 8)$. An optimized application might favor values of $\mu$

TABLE II. Damping constant $\gamma\left(\mathrm{sec}^{-1}\right)$.

\begin{tabular}{ll}
\hline \hline Silver & $2.6 \times 10^{13}$ \\
Gold & $3.7 \times 10^{13}$ \\
Copper & $4.0 \times 10^{13}$ \\
\hline \hline
\end{tabular}

TABLE I. Guide diameter examples.

\begin{tabular}{lccc}
\hline \hline Wave source & Wavelength $\lambda$ & "Standard" guide $(2 b)$ & Synchronous $\left(2 b_{\text {syn } 0}\right)$ \\
\hline$X$ band rf & $4 \mathrm{~cm}$ & $4.4 \mathrm{~cm}$ & $4.3 \mathrm{~km}$ \\
$\mathrm{CO}_{2}$ laser & $10.6 \mu \mathrm{m}$ & $12 \mu \mathrm{m}$ & $0.6 \mathrm{~mm}$ \\
Ti-sapphire laser & $0.8 \mu \mathrm{m}$ & $0.9 \mu \mathrm{m}$ & $3.5 \mu \mathrm{m}$ \\
\hline \hline
\end{tabular}


comparable to unity, as will be discussed later. The effect of finite $\mu$ on the analysis is addressed in Appendix B.

Solving the real and imaginary parts of Eq. (12) leads to expressions for the two key quantities $\left(\omega / c k_{R}-1\right)$ and $\left(k_{I} / k_{R}\right)$. The former is proportional to the dephasing rate, i.e., the rate a particle moving at $c$ slips out of phase with the wave, and the latter is related to the wave damping. This is seen from the complex phasor in the electric fields, $\exp [i(k z-\omega t)]=\exp \left[-k_{I} z+i\left(k_{R} z-\omega t\right)\right]$. Clearly $\omega / k_{R}$ is the phase velocity of the wave and $1 / k_{I}$ is the damping length. The solution for these two key quantities in the $|\mu| \ll 1$ approximation is

$$
\begin{gathered}
\omega / c k_{R}-1=\frac{\lambda_{p 2}^{2}}{\lambda^{2}} \frac{b_{\mathrm{syn} 0}^{2}}{b^{2}}\left(1-\frac{b_{\mathrm{syn} 0}}{b} f_{1}-\frac{\lambda_{p 2}^{2}}{2 \lambda^{2}} \frac{b_{\mathrm{syn} 0}^{4}}{b^{4}} f_{1}^{2} f_{2}^{2}\right), \\
1 / k_{I}=\frac{\lambda^{3}}{2 \pi \lambda_{p 2}^{2}} \frac{b^{3}}{b^{3}{ }_{\mathrm{syn} 0}}\left(f_{1} f_{2}\right)^{-1} .
\end{gathered}
$$

The factor appearing in Eq. (15), $\lambda_{p 2}^{2} / 2 \lambda^{2}$, is usually quite small, e.g., for a copper guide and a $\mathrm{CO}_{2}$ laser it is $6 \times 10^{-5}$.

Equations (15) and (16) give rise to three key length scales of the slow-waveguide mode: the channel size for perfect synchronism $b_{\text {syn }}$, the damping length $1 / k_{I}$, and the dephasing distance $L_{\mathrm{ph}}$. The synchronous channel size $b_{\text {syn }}$ is found by solving Eq. (15) for the case $\omega / c k_{R}=1$,

$$
b_{\mathrm{syn}}=b_{\mathrm{syn} 0} \cdot f_{1}\left[1-\frac{\lambda_{p 2}^{2}}{2 \lambda^{2}} \frac{f_{2}^{2}}{f_{1}^{2}}+O\left(\lambda_{p 2}^{4} / \lambda^{4}\right)\right] .
$$

Since $\lambda_{p 2} / \lambda$ is quite small, $b_{\text {syn }} \approx b_{\text {syn } 0} f_{1}$, where $b_{\text {syn } 0}$ is the undamped result, i.e., Eq. (10). The damping length was given already by Eq. (16). The dephasing distance $L_{\mathrm{ph}}$ is the distance in which the wave phase slips by $\pi$ relative to a particle moving at $c, L_{\mathrm{ph}}=\lambda / 2\left|1-c k_{R} / \omega\right|$, where $\omega / c k_{R}-1$ is a measure of the dephasing rate. In the synchronous case $\omega / c k_{R}=1$ so that the dephasing distance is infinite. For channel sizes that differ from the synchronous value in Eq. (17), the dephasing distance using Eq. (15) is

$$
L_{\mathrm{ph}}=\frac{\lambda^{3}}{2 \lambda_{p 2}^{2}}\left(\frac{b}{b_{\mathrm{syn}}}\right)^{2}\left|1-\frac{b_{\mathrm{syn}}}{b}+O\left(\lambda_{p 2}^{2} / \lambda^{2}\right)\right|^{-1} .
$$

For channels larger than the synchronous size, $L_{\mathrm{ph}}$ has a minimum of $27 \lambda^{3} / 8 \lambda_{p 2}^{2}$ at $b=(3 / 2) b_{\mathrm{syn} 0}$. Thus, for the $\mathrm{CO}_{2}$ laser wavelength and a copper boundary, the minimum value of $L_{\mathrm{ph}}$ is $29 \mathrm{~cm}$. However, $b>b_{\mathrm{syn} 0}$ causes an electric field minimum on the axis. Of more interest is the case $b<b_{\text {syno }}$ because it has a peaked field on axis. In this case, if the minimum tolerable $L_{\mathrm{ph}}$ is specified, the minimum allowed $b$ can be found using Eq. (18).

Table III shows the length scales for a cylindrical guide made of a noble metal. Small, but reasonable size microchannels support the slow-waveguide mode. The synchro-
TABLE III. Length scales for cylindrical metal guides $(\lambda=$ $10.6 \mu \mathrm{m})$.

\begin{tabular}{lccc}
\hline \hline $\begin{array}{c}\text { Wall } \\
\text { material }\end{array}$ & $\begin{array}{c}b_{\text {syn }} \\
(\mu \mathrm{m})\end{array}$ & $\begin{array}{c}b_{\min }(\mu \mathrm{m}) \\
\text { for } L_{\mathrm{ph}} \geq 10 \mathrm{~cm}\end{array}$ & $\begin{array}{c}1 / k_{I} \\
(\mathrm{~cm})\end{array}$ \\
\hline Silver & 300 & 237 & 18.4 \\
Gold & 298 & 235 & 12.8 \\
Copper & 297 & 234 & 11.8 \\
\hline \hline
\end{tabular}

nous channel diameter is $2 b \sim 600 \mu \mathrm{m}$, i.e., more than half a millimeter. Furthermore, the dephasing rate is somewhat insensitive to channel size. The damping lengths (synchronous channel) exceed $10 \mathrm{~cm}$.

\section{APPLICATION TO LASER-PARTICLE ACCELERATION}

\section{A. Relationship to other acceleration techniques}

Laser-particle acceleration is a promising application of the laser-driven metal microchannel, as illustrated in Fig. 1. Before discussing this possibility, it is helpful to note where it fits within the family of various laser acceleration techniques. The microchannel accelerator is characterized by being (i) structure based: the metal microchannel wall plays an essential role in controlling the wave speed. (ii) Near-field: the slow-wave mode is effectively a surface wave governed largely by the dielectric properties of the boundary material. (iii) Vacuum acceleration: there is no plasma or gaseous medium in the path of the particle beam. (iv) Traveling wave: the wave that accelerates the particles is launched from one end of the channel and travels with the particle beam. (v) Direct excitation: the wave is launched directly by a laser focused into one end of the channel. No nonlinear mechanism is invoked to convert laser light into another wave with suitable properties.

Microchannel acceleration has several advantages: (i) competitive gradient. The acceleration gradient is in the attractive range of $>1 \mathrm{GV} / \mathrm{m}$ for state-of-the-art $\mathrm{CO}_{2}$ lasers, as will be shown in Sec. III B. (ii) Simple physics: the linear propagation of a simple wave produces the acceleration without recourse to nonlinear waveconversion phenomena. This avoids controllability issues that often accompany nonlinear processes. (iii) Simple geometry: a smooth metal microchannel is the simplest of all slow-wave structures. A by-product of this may be less sensitivity to wall damage. (iv) Simple drive: the wave is launched by direct injection of laser light into the guide. There is no resonance condition requiring a short laser pulse. (v) Laser channeling: the metal guide automatically channels the laser beam, which can be over many Rayleigh ranges. (vi) Dephasing: for proper channel size there is perfect synchronism, i.e., no dephasing of accelerated particles. Thus the interaction length is limited only by wave damping. (vii) Electron-beam (e beam) 
quality. In the synchronous channel, the accelerating field is "flat" in the transverse direction, which helps preserve a monoenergetic $e$ beam.

The metal microchannel accelerator is related in some ways to concepts proposed earlier. The plasma fiber accelerator [5-7] employs a very narrow channel; however, the channel structure is composed entirely of plasma. The plasma at the channel edge is overdense. A variation of this assumes a rippled plasma structure so that it will support a slow wave. Another variation of this uses the beats between two waveguide modes that differ by the plasma frequency, giving the beat wave a phase velocity of $c$.

Another related concept is the hollow plasma channel [3] where the channel wall is a plasma instead of a metal. In this concept the laser beam, which is confined by the plasma channel, excites a plasma wave in the boundary plasma by forward-Raman scattering. As such this is a LWFA concept. The plasma wave is, in fact, the slowwave mode discussed here in Sec. II. It differs from the metal microchannel in that the channel is formed by a plasma instead of a metal. It also differs in that a nonlinear coupling mechanism is required to convert laser energy into the plasma wave.

A third concept is the dielectric-loaded waveguide [8]. Here the solid waveguide wall is lined with a high- $\varepsilon$ dielectric material that slows down the phase velocity of a waveguide mode to the speed of light. While the examples in Ref. [8] employ macroscopic size waveguides, a microchannel version of this has also been proposed [9]. In the example presented in Ref. [9] the channel radius is $R \sim 250 \mu \mathrm{m}$ and the index of refraction of the dielectric boundary material is $n_{2}=\left(\varepsilon_{2}\right)^{1 / 2}=1.5$, i.e., $n_{2}>1$.

\section{B. Microchannel acceleration performance}

The laser-energized metal microchannel seems ideally suited for $\mathrm{CO}_{2}$ lasers, which are capable of terawatt peak power levels [10]. The $10.6 \mu \mathrm{m}$ laser wavelength fits into a fortuitous range of frequencies suited for acceleration. If the wavelength is too short then the synchronous channel size is too small to contain a realistic-sized particle beam over a reasonable distance. As an example, for the channel diameter to exceed $100 \mu \mathrm{m}$, the laser wavelength must be $>4 \mu \mathrm{m}$ [see Eq. (10)]. Moreover, if the wavelength is too long, then the channel size becomes so large that the laser intensities on axis are much reduced. For example, if the channel diameter must be smaller than $1 \mathrm{~cm}$, then $\lambda<120 \mu \mathrm{m}$ is required.

The acceleration gradient of the microchannel accelerator can be derived by relating the accelerating field on the channel axis, $G=E_{z}(r=0)$, to realistic parameters. From Eq. (3) the radial field at the wall is $\left|E_{r W}\right|=$ $G k I_{1}\left(a_{1} b\right) / \alpha_{1}$. With $k=2 \pi / \lambda$ and synchronism $\left(\alpha_{1} b \approx\right.$ $0)$ assumed, this gives $\left|E_{r W}\right|=(\pi b / \lambda) G$. We can identify $E_{r W}$ with the laser field $E_{L}$. Thus, the acceleration gra- dient for the synchronous case $(\mu=0)$ is

$$
G \approx(1 / \pi) E_{L} \lambda / b
$$

In terms of the normalized laser field $a_{0}=e E_{L} / \omega m_{e} c$, this can be rewritten $G=2\left(m_{e} c^{2} / e\right) a_{0} / b$. The powerfield relationship for a synchronous slow wave $\left(E_{r} \propto\right.$ $r, E_{z}=$ const) is

$$
P_{L}=(\pi / 4) \varepsilon_{0} c E_{r W}^{2}\left(b^{2}+2 \lambda^{2} / \pi^{2}\right) .
$$

Combining these and recognizing that $b \gg \lambda / \pi$ gives the acceleration gradient

$$
G \approx 2 \pi \sqrt{\frac{P_{L}}{\pi \varepsilon_{0} c}} \frac{\lambda_{p 2}^{2}}{\lambda^{3}} \cdot f_{G} .
$$

Here the factor $f_{G}(\mu)$ is a correction factor for the nonsynchronous case as explained in Appendix B. In the synchronous case $f_{G}(0)=1$.

We next apply this prediction using the parameters of the $\mathrm{CO}_{2}$ laser at the Brookhaven National Laboratory Accelerator Test Facility (ATF) [10]. The ATF laser can operate in a long-pulse (180 ps) and a short-pulse $(<10 \mathrm{ps})$ mode. These laser capabilities are listed in Table IV. The acceleration gradient is for the case of a cylindrical copper guide $(b \sim 300 \mu \mathrm{m})$ and assuming a laser peak power $P_{L}=\mathrm{W}_{L} / \tau_{L}$. The predicted gradient, even for the long-pulse laser, is adequate for a proof-ofprinciple demonstration of the microchannel accelerator concept. The short-pulse laser gradient is in the interesting $\mathrm{GV} / \mathrm{m}$ range.

A cylindrical waveguide requires a radially polarized laser beam to excite the TM slow-wave mode. Radially polarized laser beams have been used at the ATF for inverse Cerenkov acceleration experiments [11]. An improved optical design [12] for creating a radially polarized beam has also been developed, which can be scaled to handle terawatt laser levels. Note, a slab waveguide can be driven using a $\mathrm{TEM}_{01}$ mode where the polarization of one lobe of this beam is $180^{\circ}$ out of phase with the other lobe. Such a TEM $_{01}$ mode is already created as an intermediate step in the schemes $[11,12]$ used to generate a radially polarized beam. Hence, a subsystem of the radially polarization conversion systems can be used to drive a slab waveguide.

The example in Table IV assumes the synchronous case for which the channel radius is $297 \mu \mathrm{m}$, the dephasing distance is infinite, the wave damping length is $11.8 \mathrm{~cm}$,

TABLE IV. Predicted gradient for ATF parameters.

\begin{tabular}{lcc}
\hline \hline \multicolumn{1}{c}{ Parameter } & $\begin{array}{c}\text { Long laser } \\
\text { pulse }\end{array}$ & $\begin{array}{c}\text { Short laser } \\
\text { pulse }\end{array}$ \\
\hline Laser pulse energy, $W_{L}$ & $5 \mathrm{~J}$ & $5 \mathrm{~J}$ \\
Laser pulse length, $\tau_{L}$ & $180 \mathrm{psec}$ & $2 \mathrm{psec}$ \\
Acceleration gradient, $G$ & $140 \mathrm{MV} / \mathrm{m}$ & $1.3 \mathrm{GV} / \mathrm{m}$ \\
\hline \hline
\end{tabular}


and the gradient is $1.3 \mathrm{GV} / \mathrm{m}$. However, the synchronous case is not necessarily the optimum. For example, higher gradients can be achieved for the nonsynchronous case $\mu>0$. Consider two examples: (i) If $\mu=0.7$, then the channel radius decreases to $b=270 \mu \mathrm{m}$, the dephasing distance is $L_{\mathrm{ph}}=36 \mathrm{~cm}$, the damping length is $1 / k_{I}=$ $10 \mathrm{~cm}$, and the acceleration gradient increases to $G=$ $1.6 \mathrm{GV} / \mathrm{m}$. (ii) If $\mu=1.5$, then the channel radius reduces to $b=237 \mu \mathrm{m}$, the dephasing distance is $L_{\mathrm{ph}}=$ $11 \mathrm{~cm}$, the damping length is $1 / k_{I}=8 \mathrm{~cm}$, and the acceleration gradient increases to $G=2.3 \mathrm{GV} / \mathrm{m}$. (See Appendix B for further discussion of these examples.)

It is useful to compare the gradient predicted for the metal microchannel with that for "resonant" LWFA [13]. Consider two LWFA examples: long wavelength, $\lambda=$ $10.6 \mu \mathrm{m}$, and short wavelength, $\lambda=0.8 \mu \mathrm{m}$. The density is chosen in order to achieve an acceleration distance of $10 \mathrm{~cm}$, i.e., by setting the dephasing distance $L_{\mathrm{ph}}=$ $\lambda_{p}^{3} / \lambda^{2}=10 \mathrm{~cm}$. LWFA resonance is assumed, i.e., $\tau_{L}=$ $\lambda_{p} / 2 c$. The laser radius is set by the requirement of quasione-dimensionality of the wakefields, i.e., $r_{L}>\lambda_{p}$.

Assume the laser pulse driving both the metal microchannel and the LWFA is $W_{L}=5 \mathrm{~J}$. The laser field parameter for a Gaussian pulse is $a_{0}=4.817 \times$ $10^{-6} \sqrt{P_{L}(W)} \lambda / r_{L}$. From Ref. [13] the gradient using $a_{0}=e E_{L} / \omega m_{e} c$ is

$$
\langle G\rangle=2 \frac{m_{e} c^{2}}{e} \frac{a_{0}^{2}}{\lambda_{p} \sqrt{1+a_{0}^{2} / 2}} .
$$

This is an average gradient assuming that the particle slips by $\pi$ in phase, which is a factor of $2 / \pi$ times the peak gradient.

Table V compares the microchannel accelerator with these two LWFA examples. The microchannel example has a gradient in the $\mathrm{GV} / \mathrm{m}$ range, but it is slightly lower than the LWFA examples. Weighed against this moderate disadvantage are the following advantages of the microchannel case: the laser pulse length is unrestricted; no plasma needs to be prepared; laser channeling is automatic; and the accelerating field is flat. In addition, as mentioned previously, higher gradients in the microchannel can be achieved by operating with slightly smaller channels, corresponding to higher fields on axis, e.g., $2.3 \mathrm{GV} / \mathrm{m}$ for a $474 \mu \mathrm{m}$ channel diameter. Alternately, if the laser pulse length in the microchannel example were reduced to that in the long-wavelength LWFA example, then $G$ would rise to $3.0 \mathrm{GV} / \mathrm{m}$.

\section{OTHER ISSUES RELATED TO METAL MICROCHANNEL ACCELERATION}

The metal microchannel is a promising acceleration concept. Nonetheless, several issues need to be investigated. Some have been addressed already. For example, the wave damping is not excessive with damping lengths exceeding $10 \mathrm{~cm}$. Moreover, precision in the microchannel dimensions is not required to achieve adequate synchronism (modest dephasing). The channel size may differ from the ideal value by several tens of percent. Other issues, which are surveyed briefly here, are subjects for future study.

If the microchannel is too small then the issue of the particle-beam acceptance emerges. This amounts to the question whether a finite-emittance beam will fit cleanly into the channel opening. For conventional beam optics, the radius of a circular beam is $a=\sqrt{a_{0}^{2}+\theta_{\infty}^{2} z^{2}}$, where $a_{0}$ is the waist radius, $\theta_{\infty}$ is the asymptotic focusing angle, and $z$ is the distance from the waist. In terms of these parameters, the normalized emittance is $\varepsilon_{N}=\gamma \theta_{\infty} b_{0}$. Suppose the beam is required to be focused in a "gentle" manner so that at the ends of the waveguide $(z=L / 2)$ we have $a=a_{\max } \leq \sqrt{2} a_{0}$. Combining these gives $a_{\max }=$ $\left(\varepsilon_{N} L / \gamma\right)^{1 / 2}$. Then for typical beam parameters at the ATF [14] of $\varepsilon_{N}=2 \times 10^{-6} \mathrm{~m} \mathrm{rad}, \gamma \sim 100$, and a section length $L=10 \mathrm{~cm}$, this gives $a_{\max }=45 \mu \mathrm{m}$. This beam radius poses no difficulty in a channel with radius $b=$ $300 \mu \mathrm{m}$.

Wall damage sets an upper limit to the intensity or electric field at the waveguide wall. Using Eq. (19) and supposing that $E_{r} \gg E_{z}$ at the boundary $r=b$, the intensity there is

$$
I_{\text {wall }}=2 \pi\left(P_{L} \lambda_{p 2}^{2} / \lambda^{4}\right) f_{I}
$$

where the factor $f_{I}(\mu)$ given in Appendix B is the nonsynchronous correction. [Recall in the synchronous case $f_{I}(0)=1$.] For the synchronous example (Table IV), the wall intensity is $I_{W}=1.7 \times 10^{15} \mathrm{~W} / \mathrm{cm}^{2}$. For the two nonsynchronous examples mentioned earlier this increases slightly: for $\mu=0.7, I_{\text {wall }}=1.98 \times 10^{15} \mathrm{~W} / \mathrm{cm}^{2}$, and for $\mu=1.5, I_{\text {wall }}=2.37 \times 10^{15} \mathrm{~W} / \mathrm{cm}^{2}$. These levels are comparable to typical optical field ionization

TABLE V. Comparison of acceleration gradients.

\begin{tabular}{lccc}
\hline \hline \multicolumn{1}{c}{ Parameter } & Microchannel & LWFA@ $\lambda=10.6 \mu \mathrm{m}$ & LWFA@ $\lambda=0.8 \mu \mathrm{m}$ \\
\hline Density $n\left(\mathrm{~cm}^{-3}\right)$ & 0 & $2.2 \times 10^{16}$ & $6.97 \times 10^{17}$ \\
Radius $b$ or $r_{L}(\mu \mathrm{m})$ & 300 & 224 & 40 \\
Laser pulse length, $\tau_{L}$ & $2 \mathrm{ps}$ & $373 \mathrm{fs}$ & $67 \mathrm{fs}$ \\
Laser parameter, $a_{0}$ & 0.38 & 0.83 & 0.83 \\
Gradient, $\langle G\rangle(\mathrm{GV} / \mathrm{m})$ & 1.3 & 2.7 & 15 \\
\hline \hline
\end{tabular}


thresholds. There is also a limit associated with the wall heating resulting from absorption of wave energy. The wall heat load is $W_{L} k_{I} / \pi b$, which for the synchronous example in Table IV is $5.3 \mathrm{~J} / \mathrm{cm}^{2}$. This is comparable to the damage thresholds for metal mirrors.

It may be possible, however, to exceed conventional damage limits because of the simplicity of the structure: a simple smooth cylindrical wall. Copper blown off the wall simply recondenses back to the wall after the laser wave passes. Thus, there are no geometrical surface changes such as might be suffered by more complex slow-wave structures involving disks, irises, or even a wavy wall. However, there is still the possibility that surface roughness caused by laser damage can scatter radiation into other waveguide modes. Damage may erode the channel ends because some metal can escape out the ends. Experiments with high-voltage discharges in smooth polyethylene microchannels as small as $300 \mu \mathrm{m}$ diameter showed that ablation was minor and the shape of the capillary remained almost the same after 1000 shots [15]. If wall damage is mitigated in this way, it may be less of a limiting factor.

In the event that some plasma is blown off the metal wall during the passage of the wave, the slow-wave phase velocity may be modified. However, it need only remain stable during the passage of the $e$-beam bunch, which typically would last on the order of $1 \mathrm{psec}$. This time is very short such that even if a plasma layer appears at the wall, it should be extremely thin. For example, for a blowoff speed equal to that of a singly ionized copper atom at $10 \mathrm{eV}$, the plasma layer after $1 \mathrm{psec}$ would be about $4 \mathrm{~nm}$ in thickness, or about $10^{-5}$ of the guide diameter. Even so the effect of a plasma layer on propagation should be investigated.

The density "profile" passes through the critical density at the surface of a metal. Resonant absorption mechanisms normally appear at the critical density, which cause wave damping and other effects, as pointed out in Ref. [3]. This might also degrade the laser beam as seen in simulations of laser acceleration schemes involving overdense plasmas [16]. However since the density jumps sharply through the resonance point at the surface of a metal this effect might be mitigated.

Nonuniformities in the wall will cause radiation into parasitic modes and thus an effective wave damping. However, enhanced ablation at "high spots" may help smooth out such nonuniformities. The effect of surface roughness on attenuation has been investigated elsewhere (see Ref. [17] and references therein).

Focusing a laser with $\lambda=10.6 \mu \mathrm{m}$ into a channel opening $600 \mu \mathrm{m}$ in diameter poses no problem. However, as mentioned earlier, efficient coupling of the laser beam into the slow-wave TM cylindrical mode requires a radially polarized laser beam. While there are different means for creating the appropriate polarized laser beam, an issue that still needs to be studied is developing efficient techniques for coupling the laser beam into the desired mode at the entrance to the waveguide.

\section{CONCLUSION}

Microchannel acceleration offers the potential for laser acceleration in free space, which avoids the usage of a phase-matching medium or plasmas, it does not require ultrashort laser pulse lengths, does not invoke nonlinear mechanisms, does not require spatial termination of the laser beam, and it is based upon a relatively simple waveguide structure. It takes advantage of the negative index of refraction of metals at infrared wavelengths, thereby making $\mathrm{CO}_{2}$ lasers ideal candidates for driving the device. At comparable peak powers as other laser acceleration schemes, microchannel acceleration yields gradients of over $1 \mathrm{GeV} / \mathrm{m}$.

While the microchannel accelerator geometry analyzed in this paper is primarily for an axisymmetric cylinder, a proof-of-principle microchannel acceleration experiment could be performed by using two opposing mirrors with a small gap between them in a slab channel geometry (see Appendix A). The $e$ beam and laser beam would be sent through the gap. This illustrates the design flexibility of this scheme.

Further analysis of design issues related to the practical implementation of this technique is still needed.

\section{ACKNOWLEDGMENTS}

This work was supported by the U.S. Department of Energy, Grant No. DE-FG03-98ER41061.

\section{APPENDIX A: APPLICATION TO SLAB CHANNELS}

The foregoing analyses assumed a cylindrical guide. For a slab channel the equations are slightly different. In Maxwell's equation for the longitudinal field in a slab guide or a surface wave [analogous to Eq. (1)], the differential operator is replaced by $d^{2} / d x^{2}$. In Eq. (3a), for a slab guide the $I_{0}$ and $I_{1}$ Bessel functions are replaced by cosh and sinh. In Eq. (3b), the $K_{0}$ and $K_{1}$ Bessel functions are replaced by $\exp \left(\alpha_{1} x\right)$ and $\exp \left(-a_{1} x\right)$. For imaginary $\alpha_{1}$, the hyperbolic functions convert as follows: $\cosh \left(\alpha_{1} x\right) \rightarrow \cos \left(\left|a_{1}\right| x\right)$ and $-i \sinh \left(\alpha_{1} x\right) \rightarrow \sin \left(\left|\alpha_{1}\right| x\right)$. The slab-guide analogy to Eq. (4) is

$$
\varepsilon_{1} \frac{\tanh \left(\alpha_{1} b\right)}{\alpha_{1} b}=-\frac{\varepsilon_{2}}{\alpha_{2} b} .
$$

For imaginary $\alpha_{1}$, the quotient on the LHS of this equation becomes $\tan \left(\left|\alpha_{1}\right| b\right) /\left|\alpha_{1}\right| b$. The slab-guide analogy to Eq. (7) is the same except the factor $K_{1}(\cdots) / \mathrm{K}_{0}(\cdots)$ is absent, and the $F$ function for a slab is defined differently:

$$
F_{\text {slab }}(\mu) \equiv\left\{\begin{array}{cc}
\tan (\sqrt{\mu}) / \sqrt{\mu} ; & \mu \geq 0 \\
\tanh (\sqrt{-} \mu) / \sqrt{-\mu} ; & \mu<0
\end{array}\right\} .
$$

The small-argument expansion of this is $F_{\text {slab }} \approx$ 
$1-\mu / 3$. The factor of $1 / 2$ on the RHS of Eqs. (9) and (12) is removed. Note, the synchronous slab-guide size $b$ is half that for the cylinder in Eqs. (10) and (17). Equation (15) has an additional factor of $3 / 2$ on the RHS and an additional factor of $3 / 2$ multiplying the last term within the parentheses. Equations (16) and (18) have an additional factor of $2 / 3$ on the RHS.

One convenient aspect of the slab-guide geometry is that it can be implemented by using a pair of flat metal mirrors facing each other with a gap of separation $b$. This will be significantly easier to fabricate and assemble than a cylindrical waveguide. Indeed, this gap can also be easily tapered to maintain phase matching should the electrons gain enough energy along the microchannel that they begin to move faster than the slow wave.

\section{APPENDIX B. CORRECTIONS FOR NONSYNCHRONOUS CONDITION}

In Sec. II we assumed $|\mu| \ll 1$, i.e., near-synchronous conditions, which allowed us to simplify the analysis by using a linear expansion of $F \approx(1 / 2)(1+\mu / 8)$ for the function in Eq. (8). However, in realistic applications the optimum may occur for a value of $\mu$ that is not small, i.e., intentionally not fully synchronous. Under these conditions usage of a more exact expression for $F$ may be required, for example, a quadratic expansion. To illustrate how the value of $F$ is affected by the approximation being made, if the exact value of $\mu$ is 0.7 [i.e., based on the exact expression for $F(\mu)$ ], then the linear approximation value is $\mu=0.79$, which is $13 \%$ too high. If the exact value of $\mu$ is 1.5 , then the linear approximation gives $\mu=$ 2.06 , or $34 \%$ too high. A quadratic expansion $F=$ $(1 / 2)\left(1+\mu / 8+\mu^{2} / 48\right)$ is much more accurate in these two examples giving values of $1.3 \%$ and $5.8 \%$ too high, respectively.

The channel size for the nonsynchronous case $\mu \neq 0$ is $b=b_{\text {syn }} f_{1} / 2 F(\mu)$. Expressions for the damping length, Eq. (16), and the dephasing length, Eq. (18), are unchanged since these are expressed in terms of the ratio $b / b_{\text {syn } 0}$.

The electric field profiles in the channel are given by Eq. (3a). In the synchronous case, $\alpha_{1} \rightarrow 0$, so that the accelerating field $E_{z}$ is flat in radius and $E_{r} \propto r$. However, in the nonsynchronous case, $\mu \neq 0, \alpha_{1} \neq 0$, so that $E_{z}$ falls off toward the channel edge, and $E_{r}$ falls faster than linear in $r$. This modifies the acceleration field-power relationship by introducing the factor $f_{G}$ in Eq. (21):

$$
f_{G}=4 F^{2}(\mu)\left[\frac{16}{\mu^{2}} \int_{0}^{\sqrt{\mu}} u d u J_{1}^{2}(u)\right]^{-1 / 2} .
$$

Finally, the wall intensity-power relationship, Eq. (23), is modified by the factor

$$
f_{I} \equiv \frac{16}{\mu}\left[J_{1}(\sqrt{\mu}) F(\mu)\right]^{2}\left[\frac{16}{\mu^{2}} \int_{0}^{\sqrt{\mu}} u d u J_{1}^{2}(u)\right]^{-1} .
$$

[1] L.C. Steinhauer, in Advanced Accelerator Concepts, Santa Fe, NM, edited by P. L. Colestock and S. Kelley, AIP Conf. Proc. No. 569 (AIP, New York, 2001), p. 266.

[2] L. C. Steinhauer and W. D. Kimura, in Proceedings of the International Conference on Lasers 2001, Tucson, AZ, 2001, edited by V. J. Corcoran and T. A. Corcoran (STS Press, McLean, 2002), pp. 153-158.

[3] T. C. Chiou, T. Katsouleas, C. Decker, W. B. Mori, J.S. Wurtele, G. Shvets, and J. J. Su, Phys. Plasmas 2, 310 (1995).

[4] J. D. Jackson, Classical Electrodynamics (Wiley, New York, 1975), 2nd ed., pp. 287-288.

[5] T. Tajima, in Proceedings of the 12th International Conference On High-Energy Accelerators, edited by F.T. Cole and R. Donaldson (Fermi National Accelerator Laboratory, Batavia, IL, 1983), p. 470.

[6] E. Zaidman, T. Tajima, D. Neuffer, K. Mima, T. Ohsuga, and D. C. Barnes, IEEE Trans. Nucl. Sci. 32, 3545 (1985).

[7] D.C. Barnes, T. Kurki-Suonio, and T. Tajima, IEEE Trans. Plasma Sci. 15, 154 (1987).

[8] C. T. M. Chang and J. M. Dawson, J. Appl. Phys. 41, 4493 (1970); K.-Y. Ng, Phys. Rev. D 42, 1819 (1990); M. Rosing and W. Gai, Phys. Rev. D 42, 1829 (1990).

[9] M. Xie, in Advanced Accelerator Concepts, Baltimore, $M D$, edited by W. Lawson, C. Bellamy, and D. Brosius, AIP Conf. Proc. No. 472 (AIP, New York, 1999), p. 701.

[10] I.V. Pogorelsky, I. Meshkovsky, A. Dublov, I. Pavlishin, Yu. A. Boloshin, G. B. Deineko, and A. Tsunemi, in Proceedings of LASERS' 98, Tucson, AZ, 1998, edited by V. J. Corcoran and T. A. Goldman (STS Press, McLean, VA, 1999), p. 911.

[11] W. D. Kimura, G. H. Kim, R. D. Romea, L. C. Steinhauer, I.V. Pogorelsky, K. P. Kusche, R. C. Fernow, X. Wang, and Y. Liu, Phys. Rev. Lett. 74, 546-549 (1995).

[12] L. P. Campbell， C.E. Dilley, S.C. Gottschalk, W. D. Kimura, L. C. Steinhauer, M. Babzien, I. Ben-Zvi, J.C. Gallardo, K. P. Kusche, I.V. Pogorelsky, J. Skaritka, A. van Steenbergen, V. Yakimenko, D. B. Cline, P. He, Y. Liu, and R. H. Pantell, IEEE Trans. Plasma Sci. 28, 1143-1151 (2000).

[13] T. Tajima and J. M. Dawson, Phys. Rev. Lett. 43, 267 (1979); for an expression for the maximum acceleration gradient, see, e.g., E. Esarey, P. Sprangle, J. Krall, and A. Ting, IEEE Trans. Plasma Sci. 24, 252 (1996).

[14] W. D. Kimura, L.P. Campbell, C.E. Dilley, S.C. Gottschalk, D.C. Quimby, A. van Steenbergen, M. Babzien, I. Ben-Zvi, J.C. Gallardo, K. P. Kusche, I.V. Pogorelsky, J. Skaritka, Y. Yakimenko, D. B. Cline, P. He, Y. Liu, L. C. Steinhauer, and R. H. Pantell, Phys. Rev. ST Accel. Beams 4, 101301 (2001).

[15] D. Kaganovich, P.V. Sasorov, Y. Ehrlich, C. Cohen, and A. Zigler, Appl. Phys. Lett. 71, 2925 (1997).

[16] T. Katsouleas, J. M. Dawson, D. Sultana, and Y.T. Yan, IEEE Trans. Nucl. Sci. 32, 3555 (1985).

[17] E. Fontana and R. H. Pantell, Phys. Rev. B 37, 3164 (1988). 